(2) Open Access Full Text Article

\title{
Aging-related rotenone-induced neurochemical and behavioral deficits: role of SIRT2 and redox imbalance, and neuroprotection by AK-7
}

This article was published in the following Dove Press journal:

Drug Design, Development and Therapy

7 May 2015

Number of times this article has been viewed

\section{Xijin Wang' \\ Qiang Guan² \\ Meihua Wang' \\ Liu Yang' \\ Jie Bai' \\ Zhiqiang Yan $^{3}$ \\ Yuhong Zhang ${ }^{4}$ \\ Zhenguo Liu'}

'Department of Neurology, Xinhua Hospital Affiliated to Shanghai Jiao Tong University School of Medicine, ${ }^{2}$ Department of Neurology, Tongji Hospital, Tongji University, ${ }^{3}$ Shanghai Laboratory Animal Center, Chinese Academy of Sciences, ${ }^{4}$ Department of Neurology, Shanghai Tenth People's Hospital, Tongji University, Shanghai,

People's Republic of China
Correspondence: Xijin Wang;

Zhenguo Liu

Department of Neurology, Xinhua Hospital Affiliated to Shanghai Jiao Tong University School of Medicine, 1665 Kongjiang Road, Shanghai 200092, People's Republic of China Tel +86 I37 $7426 \quad 1539$

Email ychwjs2004@163.com; zhenguoliu20II@aliyun.com
Abstract: Aging is one of the strongest risk factors for Parkinson's disease (PD). SIRT2 has been implicated in the aging process. It is pertinent to investigate the role of SIRT2 in agingrelated dopaminergic neurotoxicity and to develop effective therapeutic strategies for PD through the use of aging animals. In this study, we observed that rotenone induced significant behavior abnormality and striatal dopamine depletion in aging rats, while it did not do so in young rats. No significant change in striatal serotonin level was observed in the aging rats after rotenone administration. There was also aging-related rotenone-induced increase in substantia nigra (SN) SIRT2 expression in the rats. In addition, there was aging-related rotenone-induced SN malondialdehyde (MDA) increase and glutathione (GSH) decrease in the rats. No significant changes in cerebellar SIRT2, MDA, or GSH levels were observed in the aging rats after rotenone administration. Striatal dopamine content was significantly inversely correlated with SN SIRT2 expression in the rats. AK-7 significantly diminished striatal dopamine depletion and improved behavior abnormality in the rotenone-treated aging rats. Furthermore, AK-7 significantly decreased MDA content and increased GSH content in the SN of rotenone-treated aging rats. Finally, the effect of AK-7 on dopaminergic neurons and redox imbalance was supported by the results from primary mesencephalic cultures. Our study helps to elucidate the mechanism for the participation of aging in PD and suggests that SN SIRT2 may be involved in PD neurodegeneration, that AK-7 may be neuroprotective in $\mathrm{PD}$, and that maintaining redox balance may be one of the mechanisms underlying neuroprotection by AK-7.

Keywords: Parkinson's disease, environmental toxin, dopamine, oxidative stress, sirtuin

\section{Introduction}

Parkinson's disease (PD) is a common and progressive neurodegenerative disease that is characterized by motor dysfunction due to decreased dopamine (DA) content in the striatum, resulting from dopaminergic neurodegeneration in the substantia nigra (SN). ${ }^{1-3}$ Accumulating evidence indicates that the cause of PD is multifactorial, involving genetic predisposition, innate characteristics of the nigrostriatal dopaminergic system in the brain, exposure of environmental toxins and immune/inflammatory factors, and aging. ${ }^{3-12}$ Aging appears to be one of the prominent and unifying risk factors for idiopathic PD. ${ }^{13-16}$ Epidemiological studies reveal that the incidence and prevalence of PD increase with advancing age, occurring in approximately $1 \%$ of people over age 65. With the development of molecular biology and further understanding of PD, increasing importance is being attached to the search for aging-related molecules involved in PD neurodegeneration and to develop effective therapeutic strategies for PD through the use of aging animals. ${ }^{1-3}$ 
Among the various environmental factors suspected to play a role in the etiopathogenesis of $\mathrm{PD}$, agrochemical exposure has been most intensely investigated in recent years. ${ }^{1,17-24}$ The discovery of the neurotoxin rotenone has provided a valuable tool into PD research. ${ }^{17-24}$ Rotenone exposure has been observed to induce parkinsonism in rodents. ${ }^{17-21}$ At present, rotenone is widely being employed to create in vitro and in vivo models of PD in rodents. ${ }^{1,17-24}$ Rotenone was also found to induce aging-related $\mathrm{SN}$ dopaminergic neurodegeneration in rats. ${ }^{22}$ However, little is known about the effect of rotenone administration on striatal DA content and motor behavior in aging animals. In the present study, rotenone was observed to induce significant behavior abnormality and striatal DA depletion in aging rats, while it did not do so in young rats. SIRT2, a mammalian sirtuin, is a cytoplasmic $\mathrm{NAD}^{+}$-dependent deacetylase. ${ }^{25}$ It is mainly expressed in the brain..$^{25,26}$ SIRT2 is an abundant neuronal protein that accumulates in the aging central nervous system (CNS) and is indicated to be associated with the aging process..$^{25,27-30}$

Interestingly, in the present study, we observed that there was aging-related rotenone-induced increase in SIRT2 expression in the SN of rats. Furthermore, striatal DA content was significantly correlated with SN SIRT2 expression in the rats. Importantly, we investigated the role of SN SIRT2 in aging-related rotenone-induced behavior abnormality and striatal DA depletion, and the potential neuroprotection and its underlying mechanism of SIRT2 modulation in PD. Because it is not easy to obtain aging animals, young animals are often used in PD research. However, PD is an aging-related neurodegenerative disease. Aging is one of the strongest risk factors for idiopathic PD. ${ }^{13-15}$ So, aging animals are more significant for PD research than are young ones. Therefore, aging rats were employed in our study.

\section{Materials and methods}

\section{Animals and treatment}

All animals were from Sino-British SIPPR/BK Lab Animal LTD (Shanghai, People's Republic of China). Male SpragueDawley rats of two different age groups were used in the present study: a young group (3 months of age) and an aging group (18 months of age). Rotenone, emulsified in sunflower oil at $0.5 \mathrm{mg} / \mathrm{mL}$, was given intraperitoneally, at $1 \mathrm{~mL} / \mathrm{kg}$ once a day for 35 days, to the rats. At the first and eighth day of rotenone treatment, some aging rats received the selective SIRT2 inhibitor AK-7 (3-(1-azepanylsulfonyl)-N(3-bromophenyl)benzamide) by intranigral injection on both sides $(1 \mu \mathrm{g} / \mathrm{day} /$ side or $5 \mu \mathrm{g} / \mathrm{day} / \mathrm{side})$. Before rotenone treatment, the rats were anesthetized with ketamine and xylazine
$(60 \mathrm{mg} / \mathrm{kg}$ and $3 \mathrm{mg} / \mathrm{kg}$, respectively, by intramuscular injection) and positioned in a stereotaxic apparatus. Then, AK-7 $(2 \mu \mathrm{g}$ or $10 \mu \mathrm{g})$ dissolved in dimethyl sulfoxide (DMSO) $(4 \mu \mathrm{L})$ or vehicle ( $4 \mu \mathrm{L}$ of DMSO) was injected into the $\mathrm{SN}$ at a flow rate of $1 \mu \mathrm{L} / \mathrm{min}$, using a $10 \mu \mathrm{L}$ Hamilton microsyringe, with $2 \mu \mathrm{L}$ of volume for intranigral injection of each side. The following coordinates were used: $-5.4 \mathrm{~mm}$ anterior-posterior; $\pm 2.1 \mathrm{~mm}$ medial-lateral; and $-7.8 \mathrm{~mm}$ dorsal-ventral. The needle was left in place for 5 minutes, to avoid reflux along the injection track, prior to being withdrawn. All experiments were conducted according to the Guideline for Animal Experimentation of Shanghai Jiao Tong University School of Medicine and the Guide for the Care and Use of Laboratory Animals published by the US National Institutes of Health (NIH]) (publication number 85-23, revised 1996). All attempts were made to minimize the number of animals used and their suffering.

\section{Behavior tests}

Rotarod and open-field tests were performed to evaluate rat behavior during the light period. The following three parts were required for the rotarod test: (1) a roller; (2) a power source used to turn the roller; and (3) four separators dividing the roller into equal-sized compartments. Animals were placed on the rod and sequentially tested at 5, 10, $15 \mathrm{rpm}$. After being trained, the rats were tested three times at each rotarod speed. The latency time to fall was measured for each test. For locomotor activity, each rat was placed in an open-field chamber made of wood covered with impermeable formica. The chamber had a white floor $(100 \times 100 \mathrm{~cm})$ divided into 25 squares of $20 \times 20 \mathrm{~cm}$, and $50 \mathrm{~cm}$-high walls. Before testing, each rat was placed in the center of the open field and habituated for 10 minutes. Rat behavior was recorded for 30 minutes. The following parameters were determined: (1) crossing number, defined as entering of another square with all four paws; and (2) rearing number, defined as rearing with and without wall contact (standing only on hind legs).

\section{Neurochemical analysis}

Biochemical analysis of neurotransmitter in the rat striata was performed using high-performance liquid chromatography (HPLC) with electrochemical detection (ECD) (HPLCECD). ${ }^{31}$ Rat striata were removed, held on ice, and weighed. Then striata were homogenized $(10 \% \mathrm{wt} / \mathrm{vol})$ through sonication, in ice-cold homogenization buffer containing perchloric acid $(0.1 \mathrm{~mol} / \mathrm{L})$ with 3,4-dihydroxybenzylamine as internal standard. Obtained samples were centrifuged at 25,000 g 
for 10 minutes at $4^{\circ} \mathrm{C}$, and supernatants were collected. DA and serotonin (5-HT) content were assayed by HPLC-ECD, equipped with a column of $5 \mu \mathrm{m}$ spherical $\mathrm{C} 18$ particles. The mobile phase consisted of $0.1 \mathrm{M}$ phosphate buffer ( $\mathrm{pH} 2.6$ ) containing $0.2 \mathrm{mM}$ octane sulfonic acid, $2.5 \%$ methanol, and $4.5 \%$ acetonitrile. The content of each neurotransmitter was expressed as ng/g equivalent striatal tissue.

\section{Western blotting}

Western blotting was performed to evaluate SIRT2 expression, as previously described. ${ }^{8}$ The $\mathrm{SN}$ and cerebellum tissue were homogenized on ice. After centrifugation, the supernatant protein content was assayed using a commercially available protein assay kit (BioRad Laboratories, Hercules, CA, USA). Sample proteins were separated by sodium dodecyl sulfate polyacrylamide gel electrophoresis. After being blocked for 1 hour with blocking solution, membranes were incubated overnight at $4{ }^{\circ} \mathrm{C}$ with rabbit polyclonal SIRT2 antibody (1:200) (Santa Cruz Biotechnology, Dallas, TX, USA). Subsequently, the membranes were washed and incubated with horseradish peroxidase-conjugated secondary antibodies for 1 hour at room temperature. The signals were finally detected using an enhanced chemiluminescence (ECL) assay kit (EMD Millipore, Billerica, MA, USA). Results were expressed as densitometric relative units, representing the ratio of density of the target protein to $\beta$-actin.

\section{Primary mesencephalic neuron/glia cultures}

Primary rat ventral mesencephalic neuron/glia cultures were prepared, as previously described. ${ }^{24}$ Briefly, ventral mesencephalic tissues were dissected from Sprague-Dawley rats (embryonic day 14) and dissociated with a mild mechanical trituration. Depending on the experimental design, dissociated cells were seeded at $5 \times 10^{5}, 2.5 \times 10^{5}$, or $1 \times 10^{5} /$ well in poly-D-lysine-coated 24-, 48-, and 96-well cell culture plates, respectively. The culture medium was minimum essential medium (MEM) supplemented with 10\% heat-inactivated fetal bovine serum (FBS) and 10\% heat-inactivated horse serum (HS), 2 mM L-glutamine, $1 \mathrm{~g} / \mathrm{L}$ glucose, $100 \mu \mathrm{M}$ nonessential amino acids, $1 \mathrm{mM}$ sodium pyruvate, $50 \mathrm{U} / \mathrm{mL}$ penicillin, and $50 \mu \mathrm{g} / \mathrm{mL}$ streptomycin. Cultures were kept at $37^{\circ} \mathrm{C}$ in a humidified atmosphere of $5 \% \mathrm{CO}_{2}$ and $95 \%$ air. For treatment, the cultures were kept in treatment medium (MEM containing 2\% FBS, 2\% HS, 1 mM sodium pyruvate, $2 \mathrm{mM}$ L-glutamine, $50 \mathrm{U} / \mathrm{mL}$ penicillin, and $50 \mu \mathrm{g} / \mathrm{mL}$ streptomycin).

\section{Immunostaining and cell counting}

The cell cultures were processed for immunostaining detection, as previously described. ${ }^{24}$ After being blocked, they were incubated with the primary antibody, anti-tyrosine hydroxylase (TH, 1:1000) (Sigma-Aldrich Corp, St Louis, $\mathrm{MO}$, USA), at $4^{\circ} \mathrm{C}$ overnight. Then, they were detected using a secondary antibody (Molecular Probes; Life Technologies Corp, Carlsbad, CA) for 1.5 hours at room temperature. Cell counting was performed in duplicate and blindly. Ten representative areas per well were used for cell quantification.

\section{Assay of malondialdehyde (MDA) and glutathione $(\mathrm{GSH})$ and superoxide}

The level of MDA and GSH was determined by using a commercial assay kit (Cayman Chemical Co, Ann Arbor, MI, USA). The operation followed the manufacturer's protocol. The MDA and GSH content was determined in comparison with the standards and normalized to protein content Superoxide production was determined by measuring the SOD-inhibitable reduction of cytochrome c, as previously described. ${ }^{24}$

\section{Determination of protein concentration}

Protein content in the brain homogenate was measured by using the dye-binding method described by Bradford wherein bovine serum albumin (BSA) was used as a protein standard. ${ }^{32}$

\section{Statistical analysis}

Data were expressed as the mean \pm standard error of the mean (SEM). Differences were determined using twotailed Student's $t$-test for comparison between two groups and using an analysis of variance (ANOVA) and Bonferroni post hoc test for comparison between more than two groups. Normality of sample distribution and homogeneity of variances were tested before each ANOVA. When correlation analysis was performed, the Pearson's value was reported. Values of $P<0.05$ were accepted as statistically significant.

\section{Results \\ General assessment of animal health}

In the present study, none of the rats developed morbid health necessitating special treatment throughout the experimental period, and the survival rate was $100 \%$ in both the young and aging rats after rotenone treatment. As shown in Figure 1A, there was no significant difference in body weight between the 
A

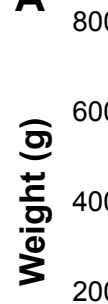

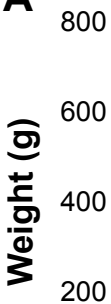

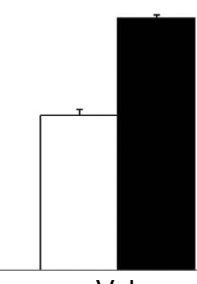

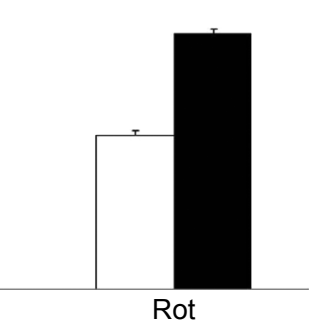

Before
B

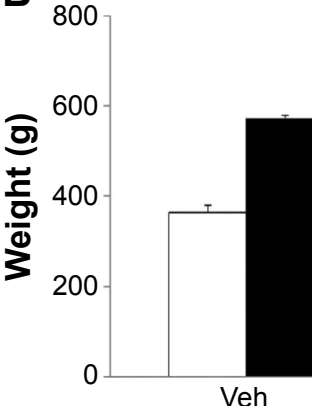

$\sqsubset$ Young

- Aging

Figure I Body weight in young and aging rats before $(\mathbf{A})$ and after $(\mathbf{B})$ Veh and Rot treatment.

Notes: Results are expressed as mean \pm SEM. $N=10$. ${ }^{\#}<0.01$ (compared with the aging rats treated with Veh).

Abbreviations: Rot, rotenone; SEM, standard error of the mean; Veh, vehicle.

young rats treated with vehicle (young-vehicle) and rotenone (young-rotenone) before treatment. Also, there was no significant difference in body weight between the aging rats treated with vehicle (aging-vehicle) and rotenone (aging-rotenone) before treatment (Figure 1A). In addition, no significant change in body weight was observed in the young-rotenone rats after rotenone treatment compared with the young-vehicle rats (Figure 1B). However, significant decrease in body weight was detected in the aging-rotenone rats after rotenone treatment compared with the aging-vehicle rats (Figure 1B) $(P<0.01)$.

\section{Aging-related rotenone-induced behavior abnormality and striatal DA depletion in rats}

Rotarod and open-field tests were performed to evaluate the effect of rotenone treatment on motor behavior in the rats. As shown in Figure 2, rotenone did not induce significant behavior change in the young rats compared with the vehicletreated rats. However, significant decreases in latency time $(P<0.005$ [5 rpm]; $P<0.01$ [10 and $15 \mathrm{rpm}])$ and number of crossings and rearings $(P<0.01$ [crossing number]; $P<0.005$
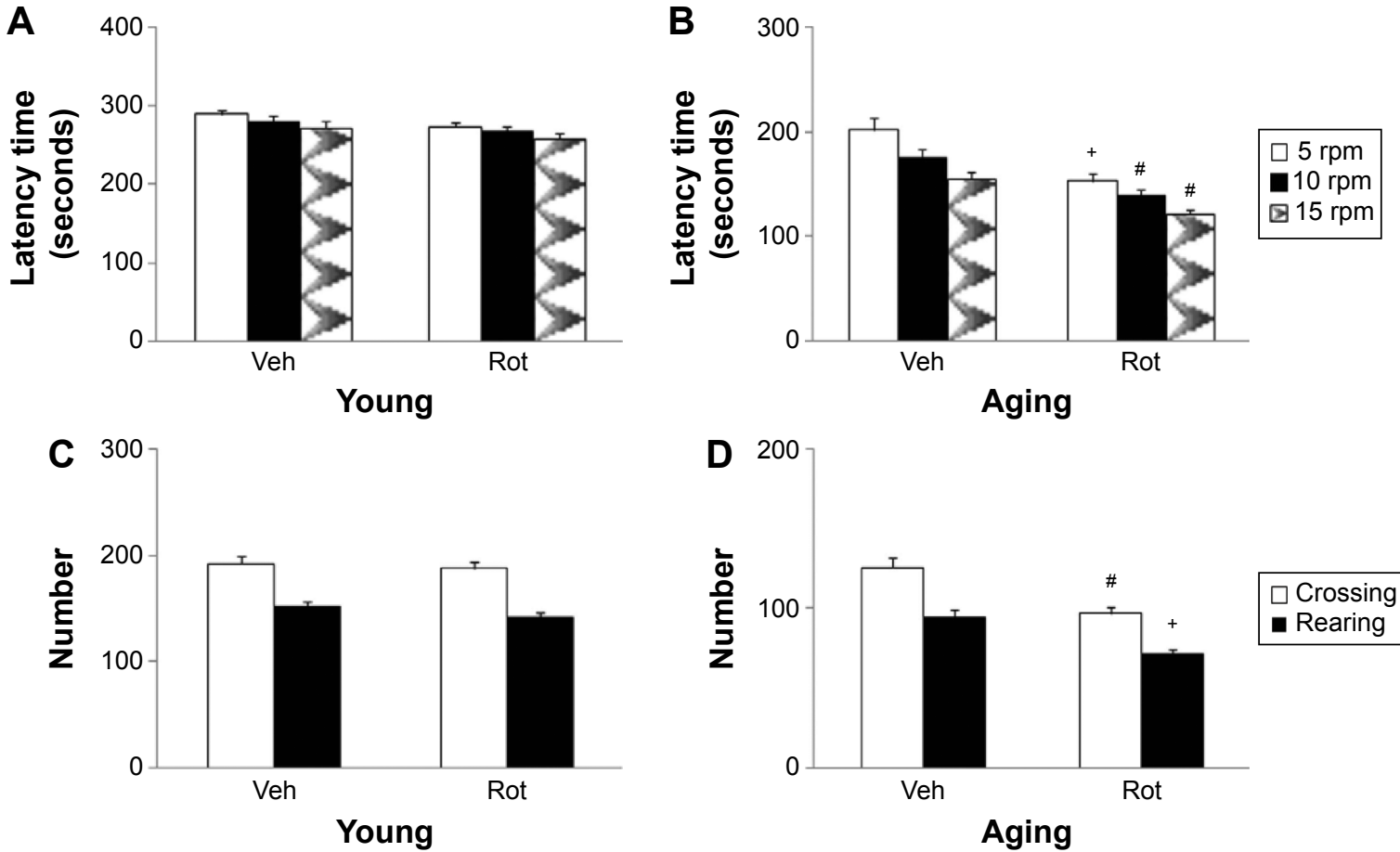

Figure 2 Effect of Rot treatment on young ( $\mathbf{A}$ and $\mathbf{C})$ and aging (B and $\mathbf{D})$ rat behavior, in the rotarod test $(\mathbf{A}$ and $\mathbf{B})$ and open-field test (C and $\mathbf{D})$.

Notes: Results are expressed as mean \pm SEM. $N=10$. ${ }^{\# P}<0.01$, compared with the aging rats treated with Veh. ${ }^{+P}<0.005$ (compared with the aging rats treated with $V$ eh). Abbreviations: Rot, rotenone; SEM, standard error of the mean; Veh, vehicle. 

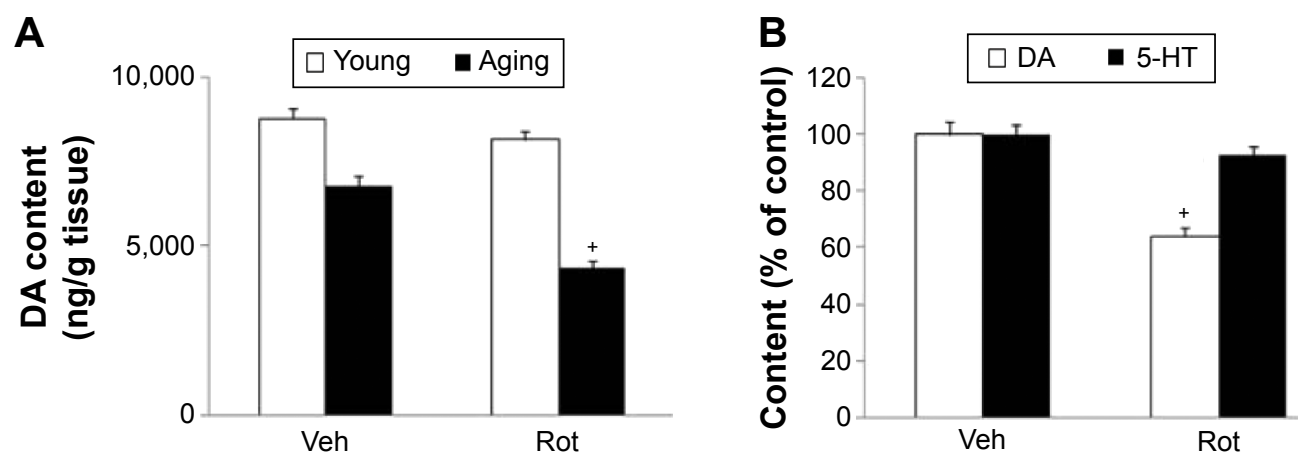

Figure 3 Effect of Rot treatment on rat striatal DA and 5-HT content in young and aging rats. (A) DA; (B) DA and 5-HT. Notes: Results are expressed as mean \pm SEM. $N=10 .{ }^{+} P<0.005$ (compared with the aging rats treated with Veh). Abbreviations: 5-HT, serotonin; DA, dopamine; Rot, rotenone; SEM, standard error of the mean; Veh, vehicle.

[rearing number]) were observed in the aging rats after rotenone treatment in comparison with the vehicle-treated rats (Figure 2). In agreement with behavior tests, rotenone did not cause significant striatal DA depletion in the young rats compared with the vehicle-treated rats (Figure 3A). However, significant decrease in striatal DA content was observed in the aging rats after rotenone treatment in comparison with the vehicle-treated rats (Figure $3 \mathrm{~A})(P<0.005)$. Although rotenone significantly decreased striatal DA content in the aging rats compared with the rats treated with vehicle, no significant change in striatal 5-HT level was observed in the aging rats after rotenone administration in comparison with the vehicle-treated rats (Figure 3B).

\section{Aging-related rotenone-induced SIRT2 expression increase in the $\mathrm{SN}$ of rats}

Western blotting was conducted to evaluate the effect of rotenone treatment on SIRT2 expression in the $\mathrm{SN}$ of rats. As shown in Figure 4A and C, rotenone did not significantly induce SIRT2 expression change in the SN of young rats compared with the vehicle-treated rats. However, significant increase in SN SIRT2 expression was observed in the aging rats after rotenone treatment in comparison with the vehicletreated rats (Figure 4B and $\mathrm{C})(P<0.005)$. In our preliminary study, rotenone $(0.1 \mathrm{mg} / \mathrm{kg})$ did not induce significant SIRT2 expression increase in the $\mathrm{SN}$ of aging rats in comparison with the rats treated with vehicle. Correlation analysis indicated that there was significant positive correlation between SN SIRT2 protein expression and the dosage of rotenone in the aging rats $(R=0.8012, P<0.005)$. Although rotenone significantly increased SN SIRT2 expression in the aging rats compared with the rats treated with vehicle, no significant change in cerebellar SIRT2 expression was observed in the aging rats after rotenone administration in comparison with the vehicle-treated rats (Figure 5A and B).

\section{Aging-related rotenone-induced MDA increase and GSH decrease in the SN of rats}

We further investigated the effect of rotenone treatment on MDA and GSH content in the SN of rats. As shown in Figure 6A, rotenone did not significantly induce MDA content change in the $\mathrm{SN}$ of young rats compared with the vehicle-treated rats. However, significant increase in SN MDA content was observed in the aging rats after rotenone treatment in comparison with the vehicle-treated rats (Figure 6A) $(P<0.005)$. In addition, rotenone did not significantly induce GSH content change in the SN of young rats compared with the vehicle-treated rats. However, significant decrease in SN GSH content was observed in the aging rats after rotenone treatment in comparison with the

A

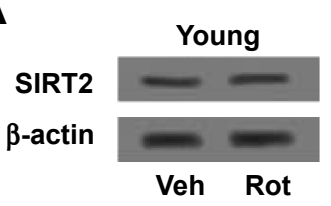

B

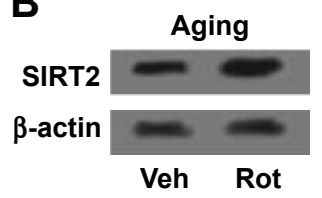

C

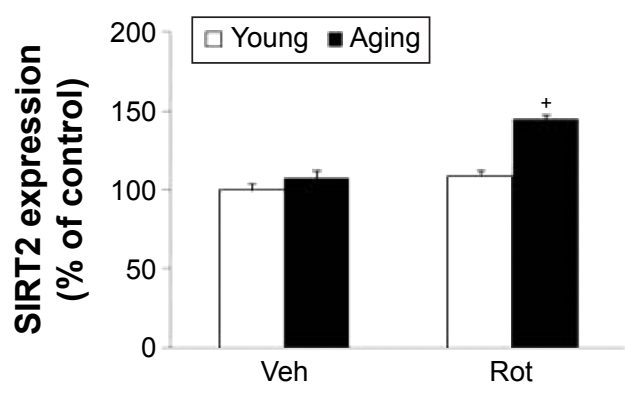

Figure 4 Aging-related Rot-induced SIRT2 expression increase in rat SN. Representative pictures of western blotting in (A) young rats and (B) aging rats. (C) The blots were quantified after correcting for $\beta$-actin.

Notes: Results are expressed as a percentage of the young rats treated with Veh (mean $\pm \mathrm{SEM}$ ). $\mathrm{N}=10 .{ }^{+} \mathrm{P}<0.005$ (compared with the aging rats treated with Veh).

Abbreviations: Rot, rotenone; SEM, standard error of the mean; SN, substantia nigra; Veh, vehicle. 
A
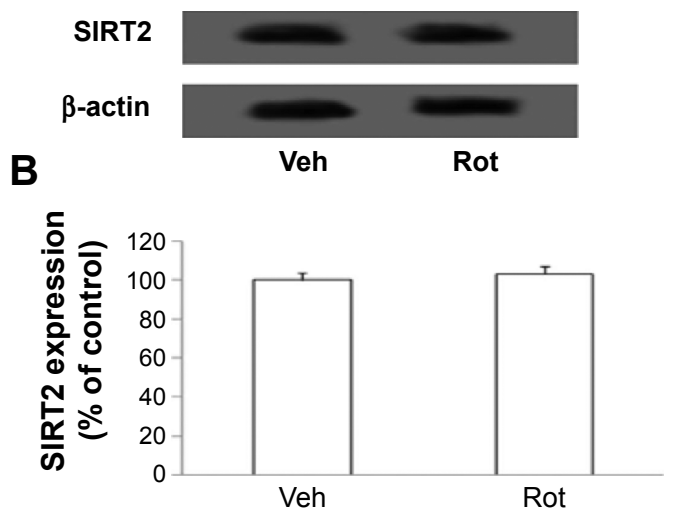

C

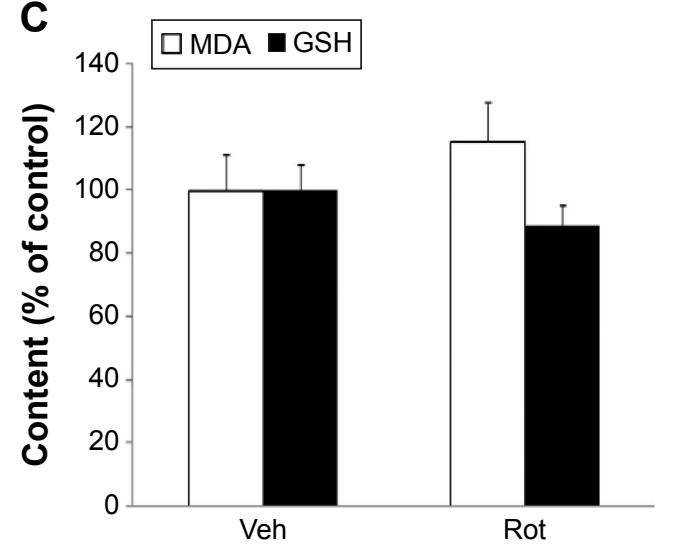

Figure 5 Effect of Rot treatment on (A and B) SIRT2 expression, and on (C) MDA and GSH content, in the CBM of aging rats. (A) representative pictures of western blotting. (B) The blots were quantified after correcting for $\beta$-actin.

Notes: Results are expressed as a percentage of the rats treated with Veh (mean $\pm \mathrm{SEM}) . \mathrm{N}=10$.

Abbreviations: CBM, cerebellum; GSH, glutathione; MDA, malondialdehyde; Rot, rotenone; SEM, standard error of the mean; Veh, vehicle.

vehicle-treated rats (Figure 6B) $(P<0.01)$. Although rotenone significantly changed SN MDA and GSH content in the aging rats compared with the vehicle-treated rats, no significant change in cerebellar MDA and GSH content was observed in the aging rats after rotenone administration in comparison with the vehicle-treated rats (Figure 5C).

\section{Role of SN SIRT2 and redox imbalance in aging-related rotenone-induced behavior abnormality and striatal DA depletion: neuroprotection by AK-7}

Correlation analysis indicated that there was significant inverse correlation between striatal DA content and $\mathrm{SN}$ SIRT2 protein expression in the rats $(R=-0.91843, P<0.005)$. AK-7 is a cell- and brain-permeable, selective SIRT2 inhibitor. So, AK-7 was used to further investigate the role of SIRT2 in aging-related rotenone-induced behavior abnormality and striatal DA depletion. As shown in Figure 7, although intranigral injection of AK-7 did not significantly change behavior abnormality in the aging rats treated with rotenone compared with the vehicle-treated rats at the dose of $1 \mu \mathrm{g} /$ day/side, it significantly improved behavior abnormality in the rotenone-treated aging rats at $5 \mu \mathrm{g} /$ day $/$ side $(P<0.05$ [crossing number in open-field tests]; $P<0.01$ [rotarod tests and rearing number in open-field tests]). In agreement with behavior tests, neurochemical analysis also showed that AK-7 administration significantly diminished striatal DA depletion in the rotenone-treated aging rats compared with the vehicle-treated rats (Figure 8$)(P<0.05[1 \mu \mathrm{g} /$ day/side $]$; $P<0.01[5 \mu \mathrm{g} / \mathrm{day} / \mathrm{side}])$. Furthermore, AK-7 treatment significantly decreased MDA content and increased GSH level in the rotenone-treated aging rats compared with the vehicle-treated rats (Figure 9) $(P<0.05$ [1 $\mu \mathrm{g} /$ day/side]; $P<0.01[5 \mu \mathrm{g} /$ day/side $])$.
A

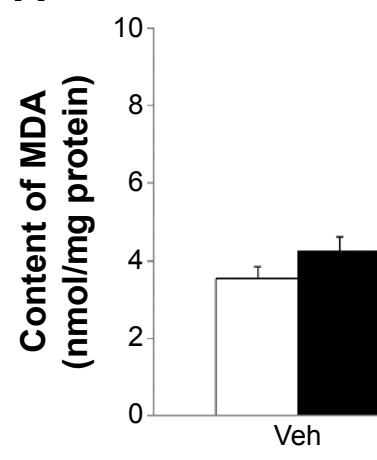

B

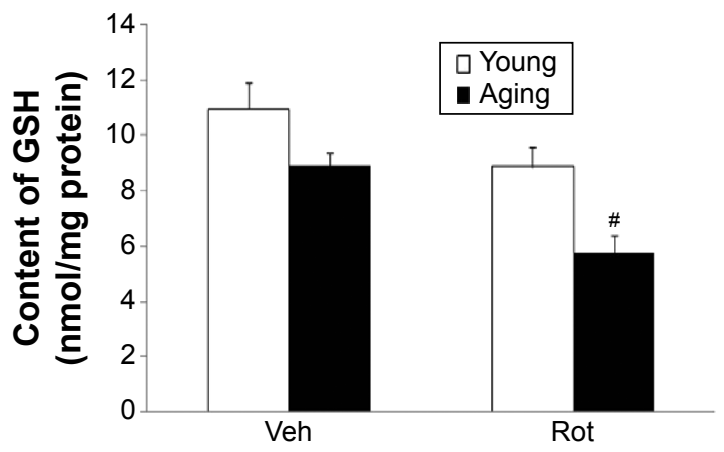

Figure 6 Effect of Rot treatment on MDA (A) and GSH (B) content in the SN of young and aging rats.

Notes: Results are expressed as mean \pm SEM. $N=10$. ${ }^{+P}<0.005$ (compared with the aging rats treated with Veh). ${ }^{\#}<0.01$ (compared with the aging rats treated with Veh). Abbreviations: GSH, glutathione; MDA, malondialdehyde; Rot, rotenone; SEM, standard error of the mean; SN, substantia nigra; Veh, vehicle. 
A

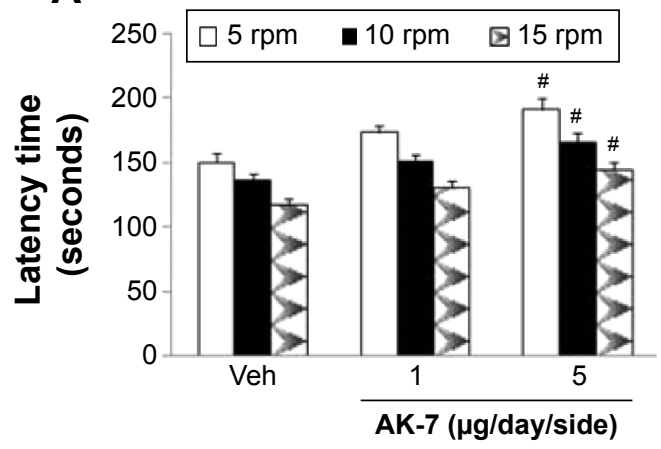

B

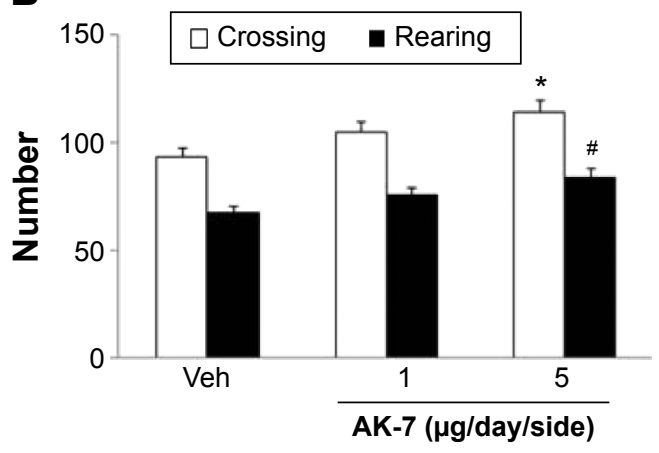

Figure 7 Effect of AK-7 treatment on motor behavior of aging rats treated with Rot in (A) the rotarod test and (B) the open-field test.

Notes: Results are expressed as mean \pm SEM. $N=10$. ${ }^{* P}<0.05$ (compared with the aging rats treated with Veh). ${ }^{*} P<0.01$ (compared with the aging rats treated with Veh). Abbreviations: Rot, rotenone; SEM, standard error of the mean; Veh, vehicle.

\section{Effect of AK-7 treatment on dopaminergic neurons in primary mesencephalic neuron/glia cultures}

To further investigate potential dopaminergic neuroprotection by AK-7, immunostaining was performed in primary mesencephalic neuron/glia cultures. As shown in Figure 10, rotenone $(10 \mathrm{nM})$ induced significant decrease in TH-immunoreactive (IR) cell number in mesencephalic cultures compared with the control cultures $(P<0.01)$. Significant increase in TH-IR (tyrosine hydroxylase-immunoreactive) cell number was observed in mesencephalic cultures cotreated with rotenone and AK-7 $(25 \mu \mathrm{M})$ in comparison with the rotenone-treated cultures $(P<0.01)$. The administration of AK-7 alone did not induce significant change in TH-IR cell number in mesencephalic cultures compared with the control cultures $(P>0.05)$. Many studies have demonstrated superoxide plays an important role in mediating dopaminergic neurodegeneration in mesencephalic cultures treated

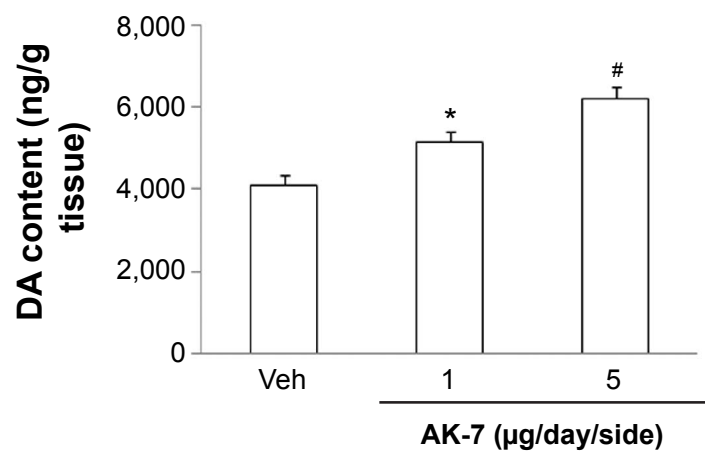

Figure 8 Effect of AK-7 treatment on striatal DA content of aging rats treated with Rot.

Notes: Results are expressed as mean \pm SEM. $N=10$. $* P<0.05$ (compared with the aging rats treated with $V$ eh). ${ }^{\#} P<0.01$ (compared with the aging rats treated with Veh).

Abbreviations: DA, dopamine; Rot, rotenone; SEM, standard error of the mean; Veh, vehicle. with neurotoxins, including rotenone. ${ }^{6,12,24}$ So, based on our in vivo results, we further investigated the effect of AK-7 administration on superoxide and GSH in mesencephalic cultures. As shown in Figure 10C, significant superoxide production decrease $(P<0.005)$ and GSH content increase $(P<0.005)$ were observed in mesencephalic cultures cotreated with rotenone and AK-7 in comparison with the rotenonetreated cultures.

\section{Discussion}

Aging is one of the strongest risk factors for idiopathic PD. ${ }^{13-15} \mathrm{PD}$ is rarely seen before 50 years of age. The incidence and prevalence of PD increase with aging. Elderly people manifest the pathological features of $\mathrm{PD}$, such as Lewy bodies, striatal DA decrease, and motor signs characteristic of PD. Phinney et al reported that rotenone $(1.5 \mathrm{mg} / \mathrm{kg} / \mathrm{day})$ treatment for 28 days did not cause significant change in the number of dopaminergic neurons in the SN of young rats; however, significant loss of SN dopaminergic neurons was observed in mature rats exposed to this same dose of rotenone. ${ }^{22}$ In the present study, we observed that rotenone treatment did not induce significant changes in striatal DA content and motor behavior in the young rats. However, significant striatal DA depletion and behavior abnormality were observed in the aging rats exposed to this same dose of rotenone. Our data presented here support and extend previous findings showing that rotenone exhibits its neurodegenerative effect in an agedependent manner. ${ }^{22}$ In addition, striatal 5-HT content was not significantly affected after rotenone treatment, showing the selectivity of rotenone neurotoxicity. It is worth noting that the dosage applied in the work is lower than in regular treatments. However, experiment results are also 
A

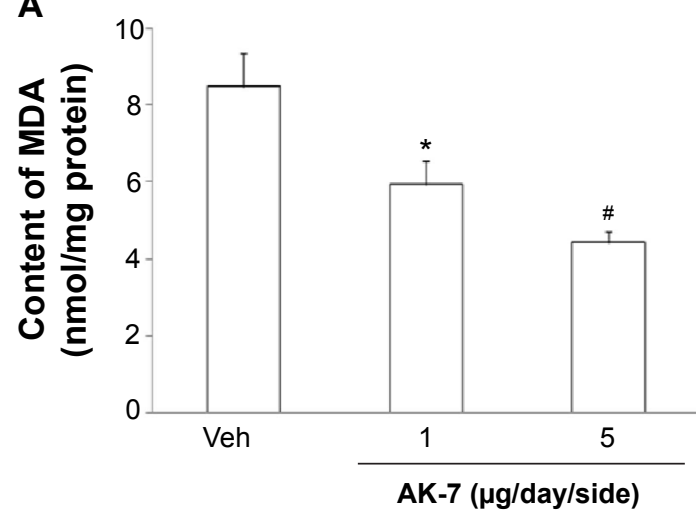

B

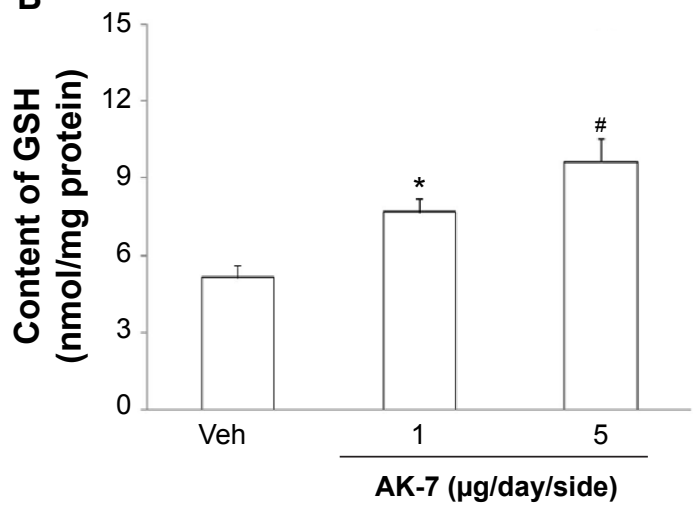

Figure 9 Effect of AK-7 treatment on MDA (A) and GSH (B) content in the SN of aging rats treated with Rot.

Notes: Results are expressed as mean \pm SEM. $N=10$. $* P<0.05$ (compared with the aging rats treated with $V$ eh). ${ }^{*} P<0.01$ (compared with the aging rats treated with $V$ eh) Abbreviations: GSH, glutathione; MDA, malondialdehyde; Rot, rotenone; SEM, standard error of the mean; SN, substantia nigra; Veh, vehicle.

determined by other factors, including age of animals and treatment period. So, it is particularly important to develop a suitable animal model for PD research. The degree of body weight loss in the aging rats treated with rotenone may suggest that aging also enhances the sensitivity of peripheral cells to rotenone. However, none of the rats developed obvious health problems in the present study. So, the toxic threshold for rotenone may be higher in the periphery than in the CNS..$^{22,33}$ Weight loss may be, at least partially, associated with reduced DA function. ${ }^{22,33}$ In our study, pulsatile treatment of rotenone was used because it may be more similar to the exposure in normal life than other administrations of rotenone. ${ }^{20}$
The sirtuins are a family of $\mathrm{NAD}^{+}$-dependent enzymes with homology to the yeast SIR2 protein. ${ }^{34,35}$ Increasing evidences indicate that sirtuins are involved in the regulation of a variety of biological activities, including chromosomal stability, transcriptional silencing, cell cycle progression, apoptosis, metabolism, autophagy, growth suppression, stress response, inflammation, and tumorigenesis. ${ }^{28,34-37}$ Recent work has implicated the role of sirtuins in the aging process and aging-related neurodegenerative diseases. ${ }^{25,38-41} \mathrm{In}$ mammals, there are seven known sirtuins, SIRT $1-7 .{ }^{35}$ Among these seven sirtuins, SIRT2 is prominently expressed in the brain. ${ }^{25,38,39}$ SIRT2 expression was observed strongest in the brain, including the postnatal hippocampus. ${ }^{42,43}$ SIRT2 is

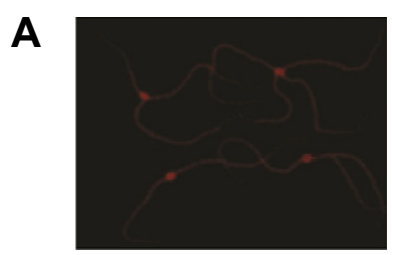

Control

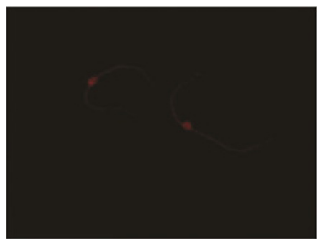

Rot

B

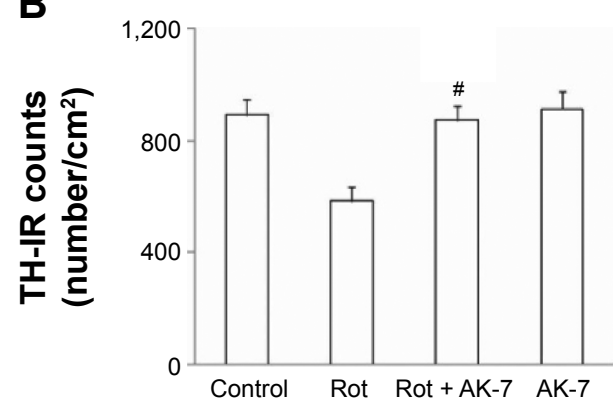

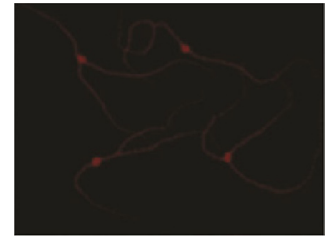

Rot + AK-7

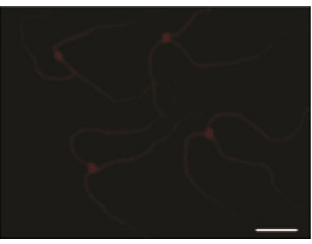

AK-7

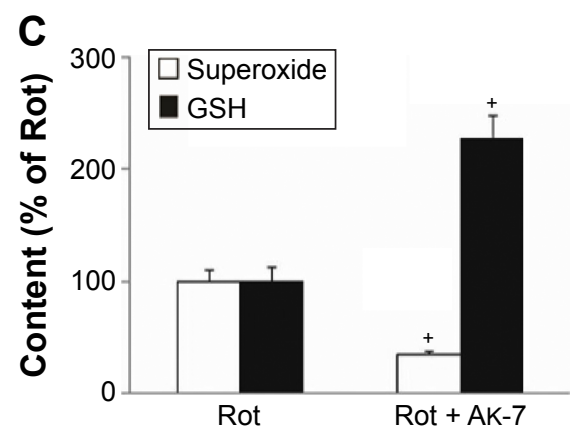

Figure 10 Effect of AK-7 treatment on (A and $\mathbf{B}$ ) dopaminergic neurons and on (C) superoxide and GSH in primary mesencephalic neuron/glia cultures. Notes: Results are expressed as mean \pm SEM. $N=12$. ${ }^{*} P<0.01$ (compared with the rotenone-treated cultures). ${ }^{+} P<0.005$ (compared with the rotenone-treated cultures). Scale bar $=100 \mu \mathrm{M}$.

Abbreviations: GSH, glutathione; MDA, malondialdehyde; Rot, rotenone; SEM, standard error of the mean; TH-IR, tyrosine hydroxylase-immunoreactive. 
rather uniformly expressed in all neurites and their growth cones in neurons. ${ }^{43}$ In neurons and oligodendrocytes, SIRT2 is confined to the cytoplasm and not the nucleus of these postmitotic cells. ${ }^{42-45}$ Outeiro et al reported that decreasing SIRT2 activity with small-molecule inhibitors, or decreasing its expression by RNA interference, ameliorated $\alpha$-synucleininduced toxicity in three different models (in vitro and in vivo): transfected human $\mathrm{H} 4$ neuroglioma cells with expression of wild-type $\alpha$-synuclein; transfected rat midbrain primary neurons expressing a variant of $\alpha$-synuclein that manifests enhanced proteotoxicity; and a Drosophila model of PD. ${ }^{46}$ The degree of rescue from $\alpha$-synuclein toxicity in $\mathrm{H} 4$ cells was comparable with that detected when Hsp70 is overexpressed. Hsp70 plays important roles in protein folding and monitors aggregation. Data from the latter two models are particularly exciting because they show decreased neuronal loss in response to reduced SIRT2 activity. Recent studies identify SIRT2 inhibition as a promising avenue for Huntington's disease therapy. ${ }^{47-49}$ In addition, SIRT2 overexpression was shown to reduce the survival of healthy neurons. ${ }^{50}$ However, the relationship of SIRT2 with PD neurodegeneration has remained unknown. Here, our results showed that there was an aging-related rotenone-induced SIRT2 expression increase in the SN of rats. SN SIRT2 protein expression was significantly positively correlated with the dosage of rotenone in the aging rats. Striatal DA content was significantly correlated with SN SIRT2 expression in the rats. It is worth noting that no significant change in cerebellar SIRT2 expression was observed in the aging rats after rotenone administration, being in best agreement with the distribution of the PD injury region, which suggests regional selectivity of the aging-related rotenone-induced change in SIRT2 expression. These results strongly indicate that there may be increased SN SIRT2 expression that is involved in PD dopaminergic neurodegeneration.

AK-7 is a selective SIRT2 inhibitor with cell and brain permeability. ${ }^{48}$ It ameliorates neuronal cell death induced by mutant huntingtin fragments. AK-7 administration was shown to result in reduced brain atrophy, extended survival, and improved motor function in two genetic mouse models of Huntington's disease. ${ }^{49}$ So, AK-7 is a good chemical probe to study the roles of SIRT2 in metabolic diseases, cancer, aging-related disorders, and neurodegenerative diseases. Here, by using AK-7, we showed that selective SIRT2 inhibition significantly diminished striatal DA depletion and improved behavior abnormality in the rotenone-treated aging rats. In addition, AK-7's dopaminergic neuroprotection in PD was further supported by the results from immunostaining in primary mesencephalic cultures. Our results suggest the role of SN SIRT2 in aging-related rotenone-induced behavior abnormality and striatal DA depletion, and the potential dopaminergic neuroprotection of AK-7 in PD.

Oxidative stress is increasingly prominent in the process of aging and neuronal cell damage. ${ }^{51-54}$ Redox imbalance may play a key role in many neurodegenerative diseases. ${ }^{55}$ Evidence of the increased oxidative stress in PD includes elevated levels of MDA, lipids, and cholesterol hydroperoxide. ${ }^{56,57}$ MDA is the most cytotoxic aldehyde produced in the process of lipid peroxidation. It reflects oxidative damage to lipids and has been found to be significantly increased in the SN of PD patients compared with other brain regions and control tissue. ${ }^{55,58,59} \mathrm{GSH}$ is an essential ubiquitous thiol tripeptide. It is an antioxidant found in all animal cells. GSH exerts cell protection by reacting with free radicals and reducing superoxide radicals, hydroxyl radicals, and peroxynitrites. GSH has also been implicated in many biological processes, including DNA synthesis and repair, protein synthesis, cellular immunity, and enzymatic reaction. ${ }^{52,55} \mathrm{GSH}$ reduction has been observed in the SN of PD patients and in the animal models of PD. ${ }^{55,60}$ In our study, rotenone was observed to induce aging-related SN MDA increase and GSH decrease in the rats. No significant changes in cerebellar MDA and GSH level were observed in the aging rats after rotenone administration. Based on the observations of MDA and GSH, we hypothesized that redox imbalance may be involved in aging-related rotenone-induced dopaminergic neurotoxicity. AK-7 could decrease the content of MDA and increase the content of GSH in the rotenone-treated aging rats. The role of recovering redox balance in AK-7's dopaminergic neuroprotection was further supported by the results from the primary mesencephalic cultures. Therefore, the recovering balance of redox in the tissue microenvironment may be one of the most likely mechanisms by which AK-7 exerted neuroprotection effects in the aging rats treated with rotenone.

\section{Conclusion}

In summary, our study helps to elucidate the mechanisms for the participation of aging in PD, and suggests that SN SIRT2 may be involved in PD neurodegeneration and that AK-7 may be neuroprotective in PD. Maintaining redox balance may be one of the mechanisms underlying neuroprotection by AK-7. Of course, we could not rule out the possibility of other factors involved in PD and aging-related neurodegeneration. Further studies, including immunostaining and cell death analysis, will bring advances to better understand the potential role of SIRT2 in aging process and PD, and to develop 
effective therapeutic strategies to slow the progression of aging and PD neurodegeneration. 29,61,62

\section{Acknowledgments}

This work was supported by the Projects of National Science Foundation of China (No. 81171204, 81171203, 30772280, 81200871, and 81200921), the Project of Shanghai Municipal Education Commission of China (No. 14YZ046), the Project of Shanghai Municipal Health and Family Planning Commission of China (No. 20134049), the Project of Shanghai Jiao Tong University of China (No. YG2013MS22), and the Projects of Shanghai Committee of Science and Technology of China (No. 11nm0503300 and 12XD1403800).

\section{Disclosure}

The authors report no conflicts of interest in this work.

\section{References}

1. Dauer W, Przedborski S. Parkinson's disease: mechanisms and models. Neuron. 2003;39(6):889-909.

2. Qian L, Flood PM, Hong JS. Neuroinflammation is a key player in Parkinson's disease and a prime target for therapy. JNeural Transm. 2010; 117(8):971-979.

3. Connolly BS, Lang AE. Pharmacological treatment of Parkinson disease: a review. JAMA. 2014;311(16):1670-1683.

4. Olanow CW, Tatton WG. Etiology and pathogenesis of Parkinson's disease. Annu Rev Neurosci. 1999;22:123-144.

5. Kidd PM. Parkinson's disease as multifactorial oxidative neurodegeneration: implications for integrative management. Altern Med Rev. 2000;5(6):502-529.

6. Gao HM, Hong JS, Zhang W, Liu B. Synergistic dopaminergic neurotoxicity of the pesticide rotenone and inflammogen lipopolysaccharide: relevance to the etiology of Parkinson's disease. J Neurosci. 2003;23(4): 1228-1236.

7. Nelson M, Huggins T, Licorish R, Carroll MA, Catapane EJ. Effects of p-Aminosalicylic acid on the neurotoxicity of manganese on the dopaminergic innervation of the cilia of the lateral cells of the gill of the bivalve mollusc, Crassostrea virginica. Comp Biochem Physiol C Toxicol Pharmacol. 2010;151(2):264-270.

8. Wang X, Chen S, Ma G, Ye M, Lu G. Involvement of proinflammatory factors, apoptosis, caspase-3 activation and $\mathrm{Ca} 2+$ disturbance in microglia activation-mediated dopaminergic cell degeneration. Mech Ageing Dev. 2005;126(12):1241-1254.

9. Wang X, Chen S, Ma G, Ye M, Lu G. Genistein protects dopaminergic neurons by inhibiting microglial activation. Neuroreport. 2005;16(3): 267-270.

10. Wang XJ, Yan ZQ, Lu GQ, Stuart S, Chen SD. Parkinson disease IgG and C5a-induced synergistic dopaminergic neurotoxicity: role of microglia. Neurochem Int. 2007;50(1):39-50.

11. Wang XJ, Liu WG, Zhang YH, Lu GQ, Chen SD. Effect of transplantation of c17.2 cells transfected with interleukin-10 gene on intracerebral immune response in rat model of Parkinson's disease. Neurosci Lett. 2007;423(2):95-99.

12. Wang XJ, Zhang S, Yan ZQ, et al. Impaired CD200-CD200R-mediated microglia silencing enhances midbrain dopaminergic neurodegeneration: roles of aging, superoxide, NADPH oxidase, and p38 MAPK. Free Radic Biol Med. 2011;50(9):1094-1106.

13. Yankner BA, Lu T, Loerch P. The aging brain. Annu Rev Pathol. 2008;3: 41-66.
14. Gureviciene I, Gurevicius K, Tanila H. Aging and alpha-synuclein affect synaptic plasticity in the dentate gyrus. J Neural Transm. 2009;116(1): $13-22$.

15. Hindle JV. Ageing, neurodegeneration and Parkinson's disease. Age Ageing. 2010;39(2):156-161.

16. Ma L, Wei L, Wu F, Hu Z, Liu Z, Yuan W. Advances with microRNAs in Parkinson's disease research. Drug Des Devel Ther. 2013;7: 1103-1113.

17. Betarbet R, Sherer TB, MacKenzie G, Garcia-Osuna M, Panov AV, Greenamyre JT. Chronic systemic pesticide exposure reproduces features of Parkinson's disease. Nat Neurosci. 2000;3(12): 1301-1306.

18. Cannon JR, Tapias V, Na HM, Honick AS, Drolet RE, Greenamyre JT. A highly reproducible rotenone model of Parkinson's disease. Neurobiol Dis. 2009;34(2):279-290.

19. Fleming SM, Zhu C, Fernagut PO, et al. Behavioral and immunohistochemical effects of chronic intravenous and subcutaneous infusions of varying doses of rotenone. Exp Neurol. 2004;187(2):418-429.

20. Alam M, Schmidt WJ. Rotenone destroys dopaminergic neurons and induces parkinsonian symptoms in rats. Behav Brain Res. 2002;136(1): 317-324.

21. Sherer TB, Kim JH, Betarbet R, Greenamyre JT. Subcutaneous rotenone exposure causes highly selective dopaminergic degeneration and alpha-synuclein aggregation. Exp Neurol. 2003;179(1):9-16.

22. Phinney AL, Andringa G, Bol JG, et al. Enhanced sensitivity of dopaminergic neurons to rotenone-induced toxicity with aging. Parkinsonism Relat Disord. 2006;12(4):228-238.

23. Moldzio R, Radad K, Krewenka C, et al. Effects of epigallocatechin gallate on rotenone-injured murine brain cultures. J Neural Transm. 2010;117(1):5-12.

24. Gao HM, Hong JS, Zhang W, Liu B. Distinct role for microglia in rotenone-induced degeneration of dopaminergic neurons. J Neurosci. 2002;22(3):782-790.

25. Dillin A, Kelly JW. Medicine. The yin-yang of sirtuins. Science. 2007; 317(5837):461-462.

26. Harting K, Knöll B. SIRT2-mediated protein deacetylation: An emerging key regulator in brain physiology and pathology. Eur J Cell Biol. 2010; 89(2-3):262-269.

27. Maxwell MM, Tomkinson EM, Nobles J, et al. The Sirtuin 2 microtubule deacetylase is an abundant neuronal protein that accumulates in the aging CNS. Hum Mol Genet. 2011;20(20):3986-3996.

28. Milne JC, Denu JM. The Sirtuin family: therapeutic targets to treat diseases of aging. Curr Opin Chem Biol. 2008;12(1):11-17.

29. Donmez G, Outeiro TF. SIRT1 and SIRT2: emerging targets in neurodegeneration. EMBO Mol Med. 2013;5(3):344-352.

30. Yoo DY, Kim DW, Kim MJ, et al. Sodium butyrate, a histone deacetylase Inhibitor, ameliorates SIRT2-induced memory impairment, reduction of cell proliferation, and neuroblast differentiation in the dentate gyrus. Neurol Res. 2015;37(1):69-76.

31. McNaught KS, Perl DP, Brownell AL, Olanow CW. Systemic exposure to proteasome inhibitors causes a progressive model of Parkinson's disease. Ann Neurol. 2004;56(1):149-162.

32. Bradford MM. A rapid and sensitive method for the quantitation of microgram quantities of protein utilizing the principle of protein-dye binding. Anal Biochem. 1976;72:248-254.

33. Baldo BA, Sadeghian K, Basso AM, Kelley AE. Effects of selective dopamine D1 or D2 receptor blockade within nucleus accumbens subregions on ingestive behavior and associated motor activity. Behav Brain Res. 2002;137(1-2):165-177.

34. Outeiro TF, Marques O, Kazantsev A. Therapeutic role of sirtuins in neurodegenerative disease. Biochim Biophys Acta. 2008;1782(6): 363-369.

35. Finkel T, Deng CX, Mostoslavsky R. Recent progress in the biology and physiology of sirtuins. Nature. 2009;460(7255):587-591.

36. Saunders LR, Verdin E. Sirtuins: critical regulators at the crossroads between cancer and aging. Oncogene. 2007;26(37):5489-5504. 
37. Kalle AM, Mallika A, Badiger J, Alinakhi, Talukdar P, Sachchidanand. Inhibition of SIRT1 by a small molecule induces apoptosis in breast cancer cells. Biochem Biophys Res Commun. 2010;401(1):13-19.

38. Longo VD, Kennedy BK. Sirtuins in aging and age-related disease. Cell. 2006;126(2):257-268.

39. Gan L, Mucke L. 2008. Paths of convergence: sirtuins in aging and neurodegeneration. Neuron. 2008;58(1):10-14.

40. de Oliveira RM, Pais TF, Outeiro TF. Sirtuins: common targets in aging and in neurodegeneration. Curr Drug Targets. 2010;11(10): 1270-1280.

41. Orozco H, Matallana E, Aranda A. Wine yeast sirtuins and Gcn5p control aging and metabolism in a natural growth medium. Mech Ageing Dev. 2012;133(5):348-358.

42. Southwood CM, Peppi M, Dryden S, Tainsky MA, Gow A. Microtubule deacetylases, SirT2 and HDAC6, in the nervous system. Neurochem Res. 2007;32(2):187-195.

43. Pandithage R, Lilischkis R, Harting K, et al. The regulation of SIRT2 function by cyclin-dependent kinases affects cell motility. J Cell Biol. 2008;180(5):915-929.

44. Li W, Zhang B, Tang J, et al. Sirtuin 2, a mammalian homolog of yeast silent information regulator-2 longevity regulator, is an oligodendroglial protein that decelerates cell differentiation through deacetylating alphatubulin. J Neurosci. 2007;27(10):2606-2616.

45. Werner HB, Kuhlmann K, Shen S, et al. Proteolipid protein is required for transport of sirtuin 2 into CNS myelin. J Neurosci. 2007;27(29): 7717-7730.

46. Outeiro TF, Kontopoulos E, Altmann SM, et al. Sirtuin 2 inhibitors rescue alpha-synuclein-mediated toxicity in models of Parkinson's disease. Science. 2007;317(5837):516-519.

47. Luthi-Carter R, Taylor DM, Pallos J, et al. SIRT2 inhibition achieves neuroprotection by decreasing sterol biosynthesis. Proc Natl Acad Sci US A. 2010;107(17):7927-7932.

48. Taylor DM, Balabadra U, Xiang Z, et al. A brain-permeable small molecule reduces neuronal cholesterol by inhibiting activity of sirtuin 2 deacetylase. ACS Chem Biol. 2011;6(6):540-546.

49. Chopra V, Quinti L, Kim J, et al. The sirtuin 2 inhibitor AK-7 is neuroprotective in Huntington's disease mouse models. Cell Rep. 2012;2(6): 1492-1497.

50. Pfister JA, Ma C, Morrison BE, D’Mello SR. Opposing effects of sirtuins on neuronal survival: SIRT1-mediated neuroprotection is independent of its deacetylase activity. PLoS One. 2008;3(12):e4090.
51. Argüelles S, Cano M, Machado A, Ayala A. Effect of aging and oxidative stress on elongation factor-2 in hypothalamus and hypophysis. Mech Ageing Dev. 2011;132(1-2):55-64.

52. Cui H, Kong Y, Zhang H. Oxidative stress, mitochondrial dysfunction, and aging. J Signal Transduct. 2012;2012:646354.

53. Sykora $\mathrm{P}$, Wilson DM, Bohr VA. Base excision repair in the mammalian brain: implication for age related neurodegeneration. Mech Ageing Dev. 2013;134(10):440-448.

54. Kamarudin MN, Mohd Raflee NA, Hussein SS, Lo JY, Supriady H, Abdul Kadir H. (R)-(+)- $\alpha$-lipoic acid protected NG108-15 cells against $\mathrm{H}_{2} \mathrm{O}_{2}$-induced cell death through PI3K-Akt/GSK-3 $\beta$ pathway

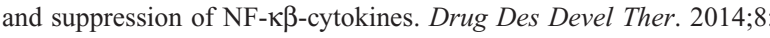
$1765-1780$

55. Navarro-Yepes J, Zavala-Flores L, Anandhan A, et al. Antioxidant gene therapy against neuronal cell death. Pharmacol Ther. 2014;142(2) 206-230.

56. Dexter DT, Carter CJ, Wells FR, et al. Basal lipid peroxidation in substantia nigra is increased in Parkinson's disease. J Neurochem. 1989; 52(2):381-389.

57. Dexter DT, Holley AE, Flitter WD, et al. Increased levels of lipid hydroperoxides in the parkinsonian substantia nigra: an HPLC and ESR study. Mov Disord. 1994;9(1):92-97.

58. Esterbauer H, Schaur RJ, Zollner H. Chemistry and biochemistry of 4-hydroxynonenal, malonaldehyde and related aldehydes. Free Radic Biol Med. 1991;11(1):81-128.

59. Ross BM, Moszczynska A, Erlich J, Kish SJ. Low activity of key phospholipid catabolic and anabolic enzymes in human substantia nigra: possible implications for Parkinson's disease. Neuroscience. 1998; 83(3):791-798.

60. Ferraro TN, Golden GT, DeMattei M, Hare TA, Fariello RG. Effect of 1-methyl-4-phenyl-1,2,3,6-tetrahydropyridine (MPTP) on levels of glutathione in the extrapyramidal system of the mouse. Neuropharmacology. 1986;25(9):1071-1074.

61. Han SH. Potential role of sirtuin as a therapeutic target for neurodegenerative diseases. J Clin Neurol. 2009;5(3):120-125.

62. Lavu S, Boss O, Elliott PJ, Lambert PD. Sirtuins - novel therapeutic targets to treat age-associated diseases. Nat Rev Drug Discov. 2008;7(10) 841-853.

\section{Publish your work in this journal}

Drug Design, Development and Therapy is an international, peerreviewed open-access journal that spans the spectrum of drug design and development through to clinical applications. Clinical outcomes, patient safety, and programs for the development and effective, safe, and sustained use of medicines are a feature of the journal, which

\section{Dovepress}

has also been accepted for indexing on PubMed Central. The manuscript management system is completely online and includes a very quick and fair peer-review system, which is all easy to use. Visit http://www.dovepress.com/testimonials.php to read real quotes from published authors. 